\title{
The PI3K inhibitor taselisib overcomes letrozole resistance in a breast cancer model expressing aromatase
}

\author{
Klaus P. Hoeflich ${ }^{1, *}$, Jane Guan ${ }^{1, *}{ }^{*}, K_{\text {Kle A. Edgar }}{ }^{1}$, Carol O’Brien $^{2}$, Heidi Savage ${ }^{2}$, \\ Timothy R. Wilson ${ }^{2}$, Richard M. Neve ${ }^{3}$, Lori S. Friedman ${ }^{1}$ and Jeffrey J. Wallin ${ }^{1}$ \\ ${ }^{1}$ Department of Translational Oncology, Genentech, Inc., South San Francisco, CA, USA \\ 2 Department of Oncology Biomarker Development, Genentech, Inc., South San Francisco, CA, USA \\ ${ }^{3}$ Department of Molecular Biology, Genentech, Inc., South San Francisco, CA, USA \\ * These authors contributed equally to this manuscript \\ Correspondence to: Jeffrey Wallin, email: jwallin@gene.com
}

Keywords: PI3K, Letrozole, Breast Cancer, GDC-0032, Akt

Received: February 29, $2016 \quad$ Accepted: March 23, $2016 \quad$ Published: March 25, 2016

This is an open-access article distributed under the terms of the Creative Commons Attribution License, which permits unrestricted use, distribution, and reproduction in any medium, provided the original author and source are credited.

\section{ABSTRACT}

Letrozole is a commonly used treatment option for metastatic hormone receptorpositive $(\mathrm{HR}+)$ breast cancer, but many patients ultimately relapse. Due to the importance of phosphoinositide-3 kinase (PI3K) in breast cancer, PI3K inhibitors such as taselisib are attractive for combination with endocrine therapies such as letrozole. Taselisib was evaluated as a single agent and in combination with letrozole in a breast cancer cell line engineered to express aromatase. The combination of taselisib and letrozole decreased cellular viability and increased apoptosis relative to either single agent. Signaling cross-talk between the PI3K and ER pathways was associated with efficacy for the combination. In a secreted factor screen, multiple soluble factors, including members of the epidermal and fibroblast growth factor families, rendered breast cancer cells non-responsive to letrozole. It was discovered that many of these factors signal through the PI3K pathway and cells remained sensitive to taselisib in the presence of the soluble factors. We also found that letrozole resistant lines have elevated PI3K pathway signaling due to an increased level of p110a, but are still sensitive to taselisib. These data provide rationale for clinical evaluation of PI3K inhibitors to overcome resistance to endocrine therapies in ER+ breast cancer.

\section{INTRODUCTION}

Breast cancer is the leading cause of nonsmoking cancer-related death in women and continues to be a major health concern [1]. Although a number of genetic and environmental factors contribute to the development of mammary epithelial neoplasia and malignancy, a wellestablished root of breast cancer is persistent exposure to endogenous or exogenous estrogen [2,3]. Anti-estrogens and aromatase inhibitors have therefore been a foundation of breast cancer treatment $[4,5]$. Tamoxifen has been the most frequently prescribed drug both as adjuvant therapy after surgery and for the treatment of advanced disease, prolonging both disease-free and overall survival. Aromatase inhibitors, such as letrozole, however, have been shown to have superior efficacy to tamoxifen in both early and advanced breast cancer with response rates of $30 \%-50 \%$ as first-line metastatic therapy [6]. However, despite these advances, endocrine therapy is limited by relapse or inevitable disease progression in the metastatic setting.

Increased signaling activity of the phosphoinositide-3 kinase (PI3K)/Akt pathway is a frequent element in most cancers [7]. Activation of the pathway occurs following activating point mutations in the PIK3CA gene (encoding the PI3K $\alpha$ isoform), which occur across the entire gene, but most frequently in the kinase and helical domains [8-10]. Genetic deletion or loss of function mutations within the tumor suppressor PTEN, a phosphatase with opposing function to PI3K, also increases PI3K pathway signaling [11]. These aberrations lead to increased downstream signaling through kinases 
such as Akt and increased activity of the PI3K pathway has been proposed as a hallmark of resistance to cancer treatment [12].

Therapeutic targeting of the PI3K pathway with small molecule inhibitors may have clinical benefit, either as single agents in PI3K-addicted cancers or used more broadly in combination with other conventional or targeted therapies. Several inhibitors targeting the PI3K pathway have now entered clinical trials [13-15]. Here we describe preclinical data for the selective PI3K inhibitor taselisib, also called GDC-0032 [16]. Taselisib potently inhibits PI3K pathway signaling and combines well with letrozole in an aromatase expressing cell line. In models of acquired letrozole resistance, we found that PI3K pathway activity was elevated, but could be blocked by taselisib. Moreover, under these conditions of acquired letrozole resistance we found the cells to be equally sensitive to taselisib. Letrozole resistant cells were subsequently cultured with increasing concentrations of taselisib to derive a model of dual resistance to endocrine/PI3K therapies. Under these conditions, the cells remained equally sensitive to taselisib

A

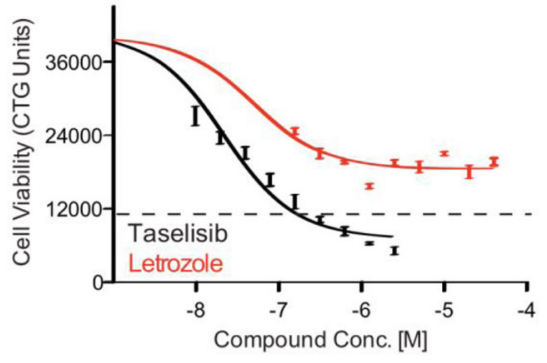

C.

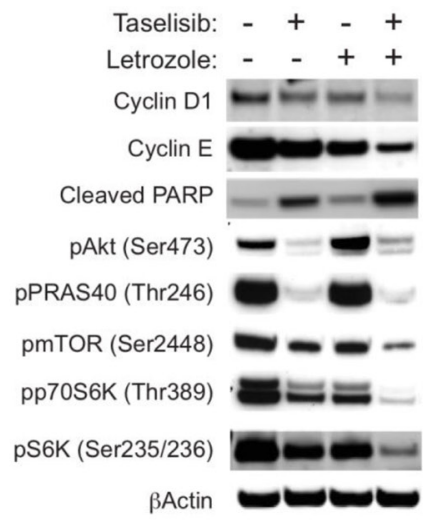

in combination with a CDK4/6 inhibitor or docetaxel. Taken together, we have developed a model to evaluate the use of PI3K and endocrine therapies in aromatase inhibitor sensitive and refractory ER+ breast cancer cells and demonstrate the activity of a novel inhibitor of PI3K in this indication.

\section{RESULTS}

\section{Combination of taselisib with letrozole decreases viability of aromatase-expressing MCF7 cells}

Taselisib, or GDC-0032, is a potent small-molecule inhibitor of class I PI3K isoforms, with reduced potency against the PI3K $\beta$ isoform and with excellent selectivity against a large panel of other kinases including closely related family members DNA-PK, VPS34, c2 $\alpha$ and c2 $\beta$ [16]. We sought to evaluate the combination effects of taselisib and letrozole in a preclinical breast cancer model

B.

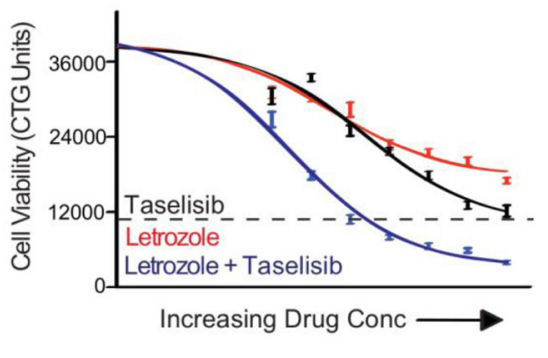

D.

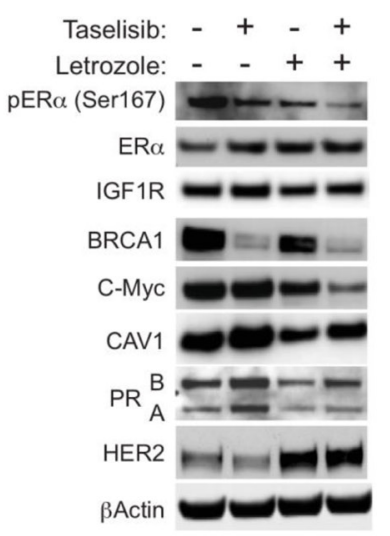

Figure 1: MCF7-ARO cells are sensitive to single agent and combination taselilsib and letrozole. (A) Cell potency of taselisib and letrozole was determined in a 96-hour viability assay. (B) Taselisib combines well with letrozole in MCF7-ARO cells. The effect on viability of taselisib and letrozole as single agents is shown in the black and red lines, respectively. The combination effect of the two drugs is indicated with the blue line. (C) Increased growth arrest and apoptosis when taselisib and letrozole are combined. Immunoblots from MCF7-ARO samples treated for 24 hours with $0.4 \mu \mathrm{M}$ taselisib and/or $0.6 \mu \mathrm{M}$ letrozole. (D) Taselisib and letrozole independently influence the expression of well-known ER target genes. Treatments are for $0.4 \mathrm{hrs}$ with $0.6 \mu \mathrm{M}$ taselisib and/or $0.1 \mu \mathrm{M}$ letrozole. Dotted lines for all viability data are indicative of CellTiterGlo counts at the beginning of drug treatment. Error bars indicate standard deviation around the mean. 
expressing aromatase. MCF7 cells were transfected with an aromatase expression construct and put under neomycin drug selection to generate stable aromataseexpressing pools (MCF7-ARO). Significant levels of estrogen were detected in supernatants of stable pool cultures after the addition of androstenedione to the media (Supplementary Figure 1a). When grown in the presence of androstenedione, MCF7-ARO cells were more reliant on estrogen for growth as evidenced by increased sensitivity to all endocrine therapies evaluated (Figure 1A

A.

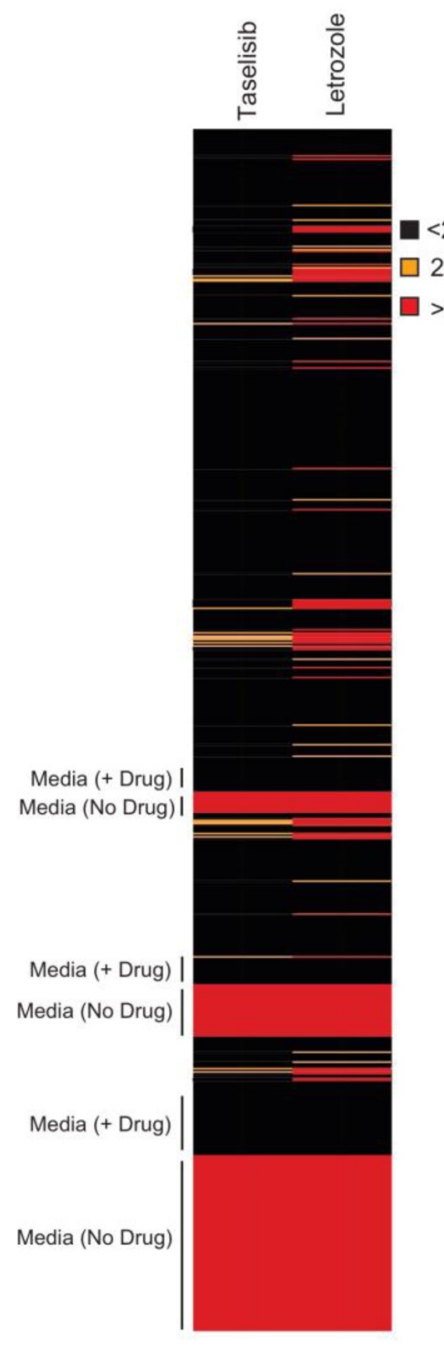

and Supplementary Figure 1B). MCF7-ARO cells were also quite sensitive to taselisib with an $\mathrm{EC}_{50}$ of $90 \mathrm{nM}$ in viability assays (Figure 1A).

MCF7-ARO cells were treated with letrozole and taselisib in a dose titration starting at $4 \mathrm{X} \mathrm{EC}_{50}$ single agent viability concentrations to determine if there is a combination effect between these two compounds. In comparison to single agent treatments, which approached $\mathrm{EC}_{50}$ levels at doses of $0.1 \mu \mathrm{M}$ for letrozole (46\% growth inhibition) and $0.1 \mu \mathrm{M}$ taselisib (47\% growth inhibition),
$<25 \%$ Rescue

25-50\% Rescue

$>50 \%$ Rescue
B.
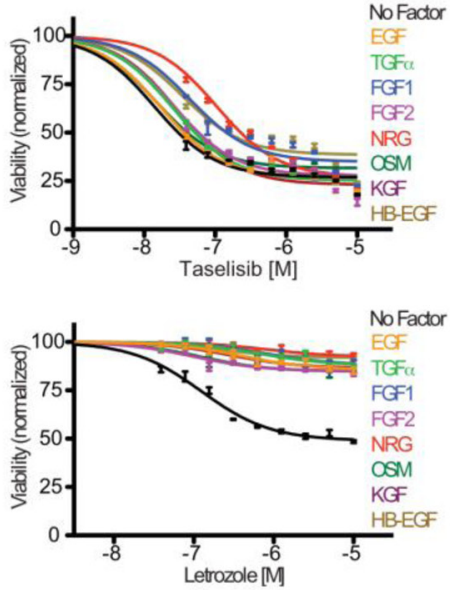

C.

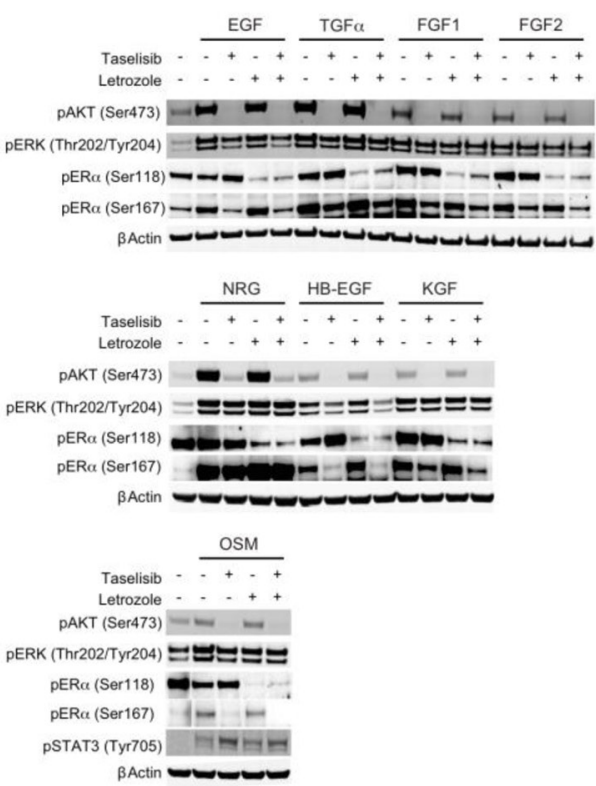

Figure 2: A subset of secreted factors mediate resistance to letrozole. (A) MCF7-ARO cells treated with DMSO, single agent taselisib or letrozole plus media or $50 \mathrm{ng} / \mathrm{ml}$ of one of 418 secreted factors, and assayed for viability using Cell-TiterGlo. Controls in the absence of secreted factors in the presence or absence of taselisib are indicated. (B) Confirmation of factors that rescue growth inhibition by letrozole. MCF7-ARO cells treated with taselisib (top) or letrozole (bottom) plus $50 \mathrm{ng} / \mathrm{ml}$ of the indicated secreted factors, and assayed for viability using CellTiter-Glo. (C) Many of the secreted factors signal down the PI3K pathway. MCF7-ARO cells simulated with 50 ng/ $\mathrm{ml}$ of the indicated secreted factors in the presence of DMSO, $0.6 \mu \mathrm{M}$ taselisib, $1 \mu \mathrm{M}$ letrozole or a combination of the two drugs. After 1 $\mathrm{hr}$, cell lysates were prepared and analyzed by immunoblotting for $\mathrm{pERK}^{\mathrm{T} 202 / \mathrm{Y} 204}, \mathrm{pAKT}^{\mathrm{S} 473}, \mathrm{pER} \alpha^{\mathrm{S} 118}, \mathrm{pER}^{\mathrm{S} 167}{ }^{\mathrm{pSTAT}}{ }^{\mathrm{Y} 705}$, and $\beta A c t i n$. For viability experiments, error bars indicate standard deviation around the mean. 
the combination of taselisib and letrozole reduced MCF7ARO viability by $81 \%$ (Figure $1 \mathrm{~B}$ ).

The effect of the compounds on downstream PI3K pathway markers was investigated with single agent and combination drug treatments at their $\mathrm{EC}_{50}$ concentrations at a 24-hour timepoint (Figure 1C). Taselisib caused a decrease of these pathway markers in the presence or absence of letrozole, as expected for an inhibitor of PI3K. We also detected an increase in phospho-Akt ${ }^{\text {Ser473 }}$ in response to letrozole alone, which has been described previously for endocrine therapy treatment [17, 18]. Letrozole treatment modulated markers at mTOR and downstream which is consistent with previous reports of estrogen regulated processes [19].

To determine the effects of drug combinations, we looked at the cell cycle and apoptotic markers - cyclin
D1, cyclin E, and cleaved poly (ADP-ribose) polymerase (PARP) after 24 hours of treatment (Figure 1C). Cyclin D1 and cyclin E are expressed in proliferating cells and help control the progression of cells through the cell cycle. Reduction of these cyclins was observed with single-agent treatments and was further decreased with combined presence of both agents. PARP is one of the main cleavage targets of caspase 3 , and cleaved PARP serves as a marker of apoptotic cells [20]. A modest increase in this marker was detected with single agents, especially with taselisib treatment, but a substantial increase in cleaved PARP was detected with the combination.

To analyze the combination effects of taselisib and letrozole on ER signaling we first evaluated ER $\alpha$ phosphorylation on serine 167, a marker of ER activation that is downstream of PI3K signaling (Figure 1D) [21,

A.
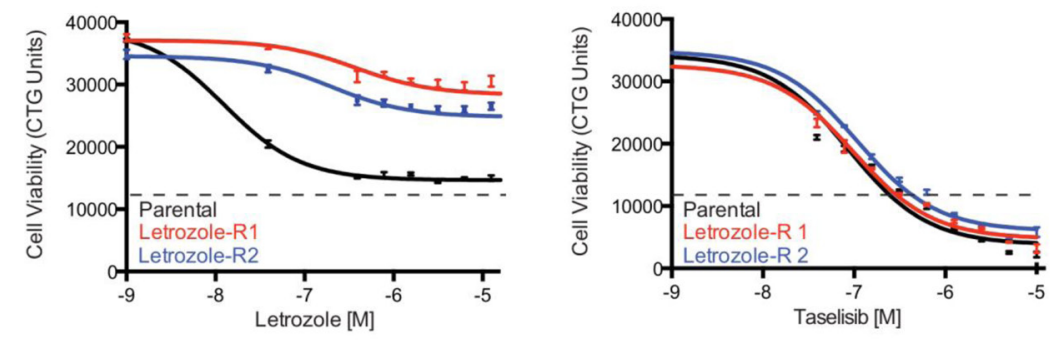

B.
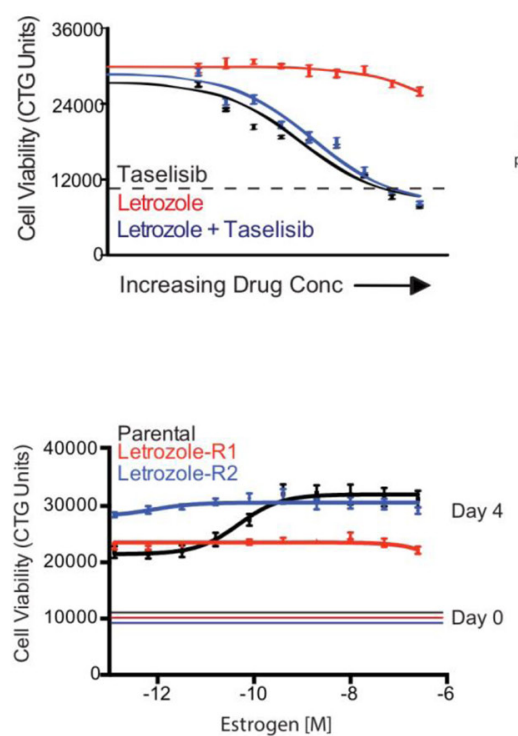

C.
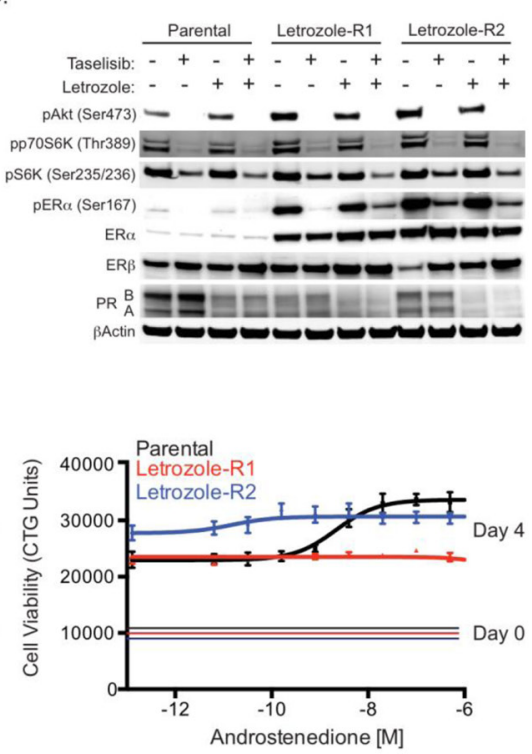

Figure 3: Characterization of letrozole resistant cells. (A) MCF7-ARO parental or letrozole resistant cells treated with a dose titration of letrozole (left) or taselisib (right) and assayed for viability using CellTiter-Glo (CTG) 96 hrs post dosing. Dotted lines are indicative of CTG counts at the beginning of drug treatment. (B) Similar response to taselisib by itself or in combination with letrozole, in letrozole resistant cells. The effect on viability of taselisib and letrozole as single agents is shown in the black and red lines, respectively. The combination effect of the two drugs is indicated with the blue line. Dotted lines are indicative of CTG counts at the beginning of drug treatment. (C) Letrozole resistant cells have increased ER $\alpha$. Treatments are for 24 hrs with $0.1 \mu \mathrm{M}$ taselisib and/or $0.1 \mu \mathrm{M}$ letrozole. (D) Resistant cells do not increase proliferation in response to estrogen. MCF7-ARO parental or letrozole resistant cells cultured with a dose titration of androstenedione (right) or estrogen (left) and assayed for viability using CellTiter-Glo after 96 hrs. Solid lines are indicative of CTG counts prior to estrogen or androstenedione treatment. Error bars indicate standard deviation around the mean. 
22]. MCF7-ARO cells were either vehicle-treated, treated with single agent taselisib or letrozole, or the combination of these agents for $24 \mathrm{hrs}$. We found that both inhibitors reduced phospho-ER $\alpha^{\text {Ser167}}$, and the combination was even more effective (Figure 1D). A small increase in total ER protein was also observed with letrozole and taselisib treatments. We also evaluated the expression of commonly described ER target genes with these treatments [23, 24, 25]. We observed a strong decrease in BRCA1 with PI3K pathway blockade that was consistent with previous reports in breast cancer models [26]. C-Myc protein, a marker downstream of both the PI3K and ER pathways was decreased with the two drugs in combination. We observed additional examples of cross-talk between the PI3K and ER pathways. Caveolin-1 (CAV1) and progesterone receptor (PGR) are two commonly described ER-regulated genes. Protein levels of these markers were decreased as expected with letrozole treatment, but these markers were increased with taselisib treatment. HER2 was increased with letrozole treatment, but not inhibition of PI3K.

\section{Multiple soluble factors that activate the PI3K pathway confer resistance to letrozole in MCF7- ARO cells}

We next investigated potential taselisib and letrozole resistance mechanisms due to factors secreted by the tumor microenvironment or other tissues. For these experiments we utilized a screen of commercially available factors to identify candidates that rescue taselisib- or letrozoleinduced growth inhibition. For the screen, MCF7-ARO cells were dosed with a $1 \mu \mathrm{M}$ concentration of taselisib or letrozole as well as $50 \mathrm{ng} / \mathrm{ml}$ of one of 418 soluble ligands for 72 hours (Supplementary Table 1). Four factors (FGF1, FGF2, KGF, and NRG) were able to rescue taselisib growth inhibition by greater than $25 \%$. Factors that

A.
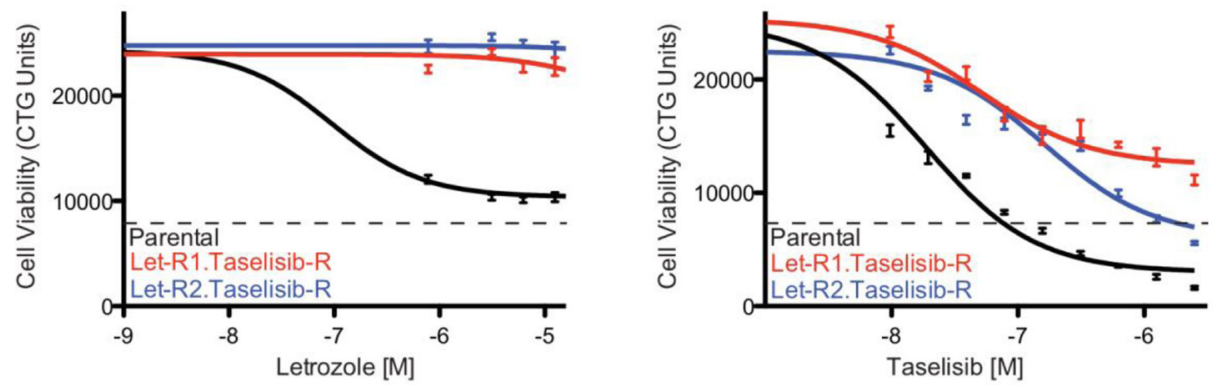

B.

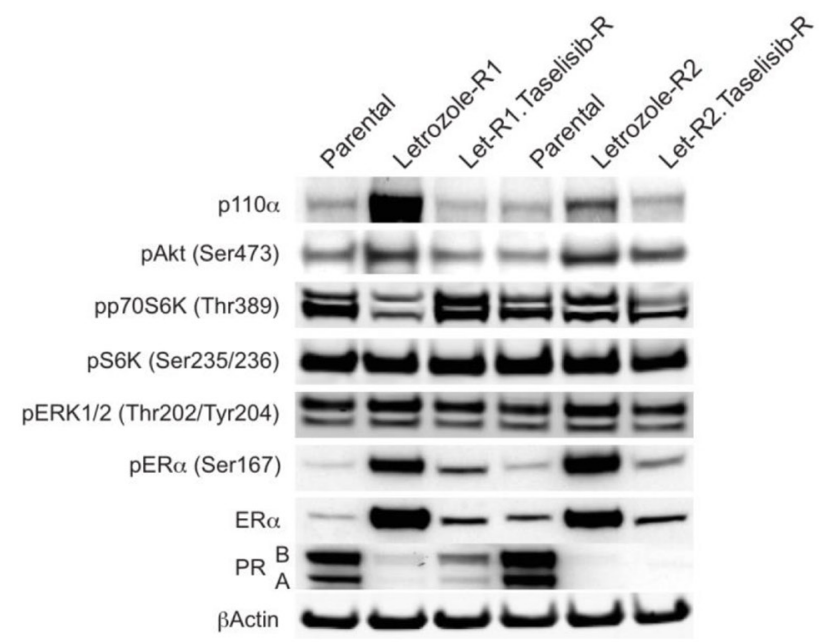

Figure 4: Characterization of cells resistant to both letrozole and taselisib. (A) MCF7-ARO parental or taselisib/letrozole resistant cells treated with a dose titration of letrozole (left) or taselisib (right) and assayed for viability using CellTiter-Glo 96 hrs post dosing. (B) Protein changes with the acquisition of resistance to letrozole or dual resistance to letrozole and taselisib, compared to parental MCF7-ARO. Cell lysates were prepared and analyzed by immunoblotting for the markers indicated. Dotted lines for all viability data are indicative of CTG counts at the beginning of drug treatment. Error bars indicate standard deviation around the mean. 
promoted resistance to letrozole were more common. We found that 26 factors $(6.2 \%$ of total $)$ were able to rescue letrozole-induced growth inhibition greater than $25 \%$ and $15(3.6 \%)$ of those factors rescued growth inhibition above $50 \%$ (Figure 2A and Supplementary Table 1). To confirm the ability of these ligands to overcome letrozole-induced growth inhibition, eight factors were tested for their effect on the cellular potency of letrozole or taselisib in MCF7ARO cells. Consistent with their activity in the large secreted factor screen, these factors promoted complete restoration of letrozole-induced growth inhibition (Figure 2B). Some of these factors also reduced the effectiveness of taselisib in viability experiments, but to a lesser degree. Inhibition was reduced approximately 2-fold by FGF1 and HB-EGF, while NRG resistance approached 3-fold.

To find the underlying mechanism of the ability of these ligands to decrease letrozole sensitivity, we investigated downstream signaling in the PI3K, MAPK and estrogen signaling pathways. All ligands increased the PI3K pathway marker $\mathrm{pAKT}^{\mathrm{Ser} 473}$ (Figure 2C).

A.
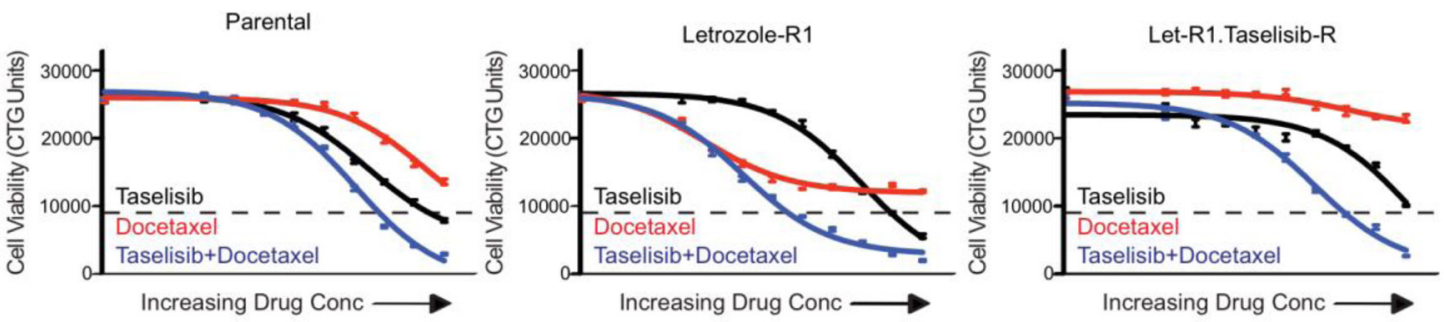

B.
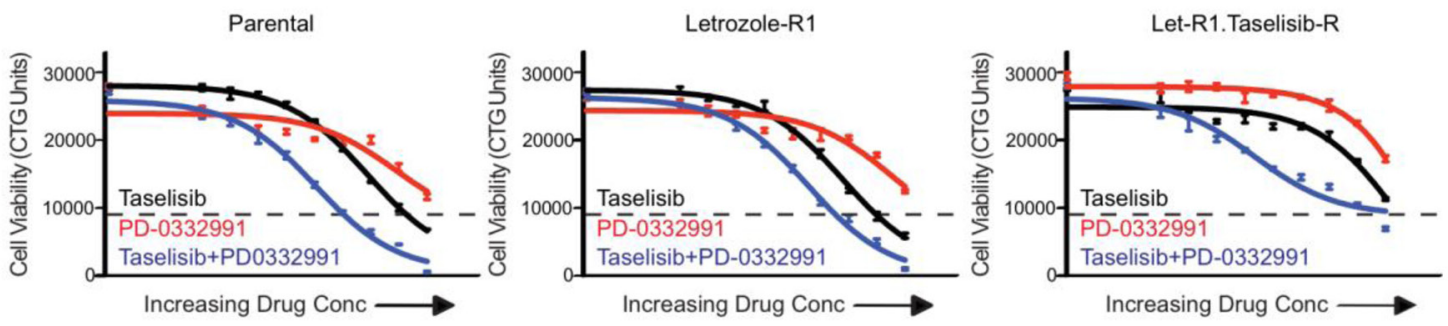

C.

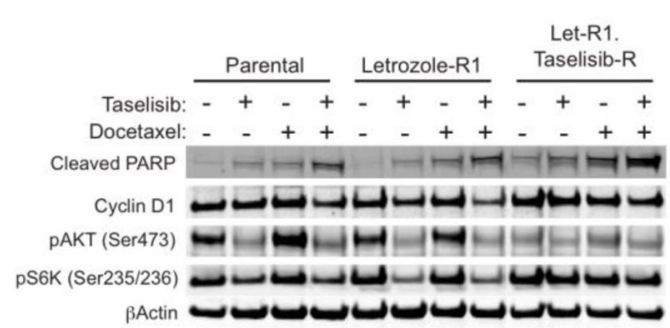

D.

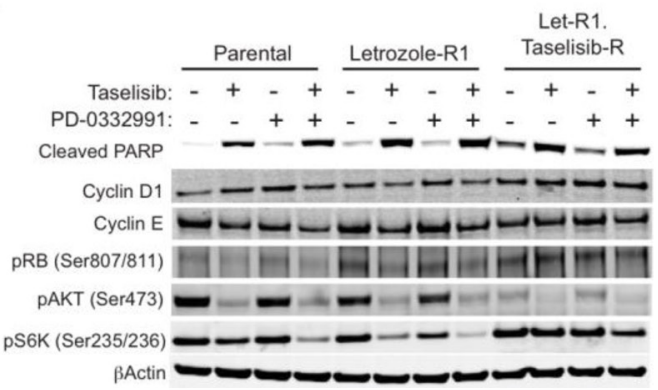

Figure 5: Dual resistant cells are still sensitive to taselisib in combination with docetaxel or CDK4/6 inhibition. (A) Taselisib combines well with docetaxel in MCF7-ARO cells. The effect on viability of taselisib and docetaxel as single agents is shown in the black and red lines, respectively. The combination effect of the two drugs is indicated with the blue line. Starting doses for taselisib were $80 \mathrm{nM}$ for the parental and letrozole-R1 lines and $10 \mu \mathrm{M}$ for taselisib for Let-R1.GDC-0032-R. Docetaxel starting doses were 36 $\mathrm{nM}$ for all three lines. (B) Taselisib combines well with PD-0332991 in MCF7-ARO cells. The effect on viability of taselisib and PD0332991 as single agents is shown in the black and red lines, respectively. The combination effect of the two drugs is indicated with the blue line. Starting doses for taselisib were $80 \mathrm{nM}$ for the parental and letrozole-R1 lines and $10 \mu \mathrm{M}$ for dual-resistant. PD-0332991 starting doses were $10 \mu \mathrm{M}$ for all three lines. (C) Increased apoptosis is observed with the taselisib and docetaxel combination in cells sensitive or resistant to taselisib and letrozole. Immunoblots from samples treated for 24 hours with $20 \mathrm{nM}$ taselisib (Parental and Letrozole-R1) or $2.5 \mu \mathrm{M}$ (Let-R1.Taselisib-R) and/or $9 \mathrm{nM}$ docetaxel. (D) Increased growth arrest is observed with combined PI3K and CDK4/6 inhibition. Immunoblots from samples treated for 24 hours with $20 \mathrm{nM}$ taselisib (Parental and Letrozole-R1) or $2.5 \mu \mathrm{M}$ (Let-R1.Taselisib-R) and/or $2.5 \mu \mathrm{M}$ PD-0332991. Dotted lines for all viability data are indicative of CTG counts at the beginning of drug treatment. Error bars indicate standard deviation around the mean. 
Letrozole treatment did not affect $\mathrm{pAKT}^{\mathrm{Ser} 473}$, but this marker was decreased in the presence of taselisib. Phospho-ERK1/2 $202 / \mathrm{Y} 204$ was increased with secreted factor treatments in the presence or absence of taselisib or letrozole, suggesting the cells could use activation of the MAPK pathway under conditions of ligand stimulation. Estrogen receptor (ER) phosphorylation provides an important mechanism to regulate ER activity [21, 27]. Two well-described phosphorylation sites on ER $\alpha$ are serine 118 and 167 , both located in the amino-terminal transcription activation domain. Increased phosphorylation of ER $\alpha$ on serine 167 was detected following stimulation with the evaluated soluble factors. Changes in $\mathrm{pER} \alpha^{\mathrm{Ser} 118}$, however, were not detected with stimulation. Signaling changes elicited by inhibitor treatment converged on two key sites of post-translational modification of estrogen receptor. Phospho-ER $\alpha^{\text {Ser118 }}$ was decreased with letrozole, while Phospho-ER $\alpha^{\text {Ser167 }}$ was decreased with taselisib treatment.

Oncostatin M (OSM) is a member of the IL-6 family of cytokines and induces activation of the Jak2/ Stat3 pathway [28]. Minor increases in $\mathrm{pAKT}^{\mathrm{Ser} 473}$ and $\mathrm{pER} \alpha \alpha^{\mathrm{Ser} 167}$ were detected with OSM stimulation that could be inhibited by taselisib (Figure 2C). We also observed increased pSTAT3 ${ }^{\mathrm{Ty} 705}$ with OSM stimulation of MCF7ARO cells that could not be blocked by taselisib or letrozole, which implies that activation of this alternative pathway may confer resistance to letrozole by this cytokine (Figure 2C).

\section{Letrozole resistant cells exhibit increased PI3K pathway signaling}

In addition to investigating the role of soluble ligands in acute or innate letrozole resistance, we sought to examine factors involved in acquired resistance to letrozole. MCF7-ARO cells were treated at increasing doses of letrozole over a period of 4 months and two resistant pools were generated. At the end of the dose escalation, the cells were able to grow at a letrozole concentration approximately 10 -fold higher $(6.25 \mu \mathrm{M})$ than the dose required for $50 \%$ growth inhibition of MCF7-ARO parental cells $(0.625 \mu \mathrm{M})$. The generated resistant pools were significantly more resistant to letrozole and other estrogen therapies, compared to the parental MCF7-ARO line (Figure 3A and Supplementary Figure 2). Taselisib sensitivity in the letrozole resistant lines, however, was similar to the parental line. In combination experiments, letrozole resistant cells were equally sensitive to single agent taselisib or letrozole in combination with taselisib (Figure 3B).

Once the resistant pools were confirmed to retain resistance to letrozole, signaling components of the PI3K and ER pathways were evaluated by western blot analysis. Both of the letrozole resistant pools were shown to have increased levels of the class I PI3K isoform p $110 \alpha$ which was not associated with increased gene expression (Supplementary Figure 3 and Figure 4b). Not surprising, we observed augmented levels of phospho-Akt at the S473 phosphorylation site in the resistant pools, a marker proximal to the PI3K enzyme in the pathway. Other PI3K pathway components did not appear to increase, but all markers of this pathway were reduced with taselisib treatment (Figure 3C).

Changes in ER $\alpha$ protein levels have been described in long-term estrogen-deprived (LTED) models of endocrine resistance [29, 30]. In LTED models with increased $\mathrm{ER} \alpha$, an augmented response to estrogen stimulation was also observed for cell growth. In our letrozole resistant MCF7-ARO cells, we also detected significantly increased $\mathrm{ER} \alpha$, which correlated with increased phospho-ER $\alpha^{\mathrm{Ser} 167}$, but decreased PR (Figure $3 \mathrm{C})$. We did not detect changes in $\mathrm{ER} \beta$ in letrozole

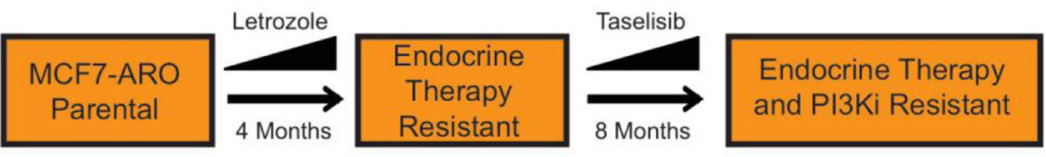

\begin{tabular}{|c|c|c|c|}
\hline $\begin{array}{l}\text { Drug } \\
\text { Sensitivity }\end{array}$ & $\begin{array}{l}\text { Letrozole } \\
\text { Taselisib }\end{array}$ & Taselisib & $\begin{array}{l}\text { Taselisib combinations } \\
\text { (Docetaxel, PD-0332991 }\end{array}$ \\
\hline
\end{tabular}

Increased: $\mathrm{p} 110 \alpha, \mathrm{pAkt}$, $\mathrm{HER} 2, \mathrm{pER} \alpha, \mathrm{ER} \alpha$

Decreased: PR

Figure 6: Underlying resistance mechanisms to letrozole and taselisib in the MCF-ARO model. Drug sensitivity and cell alterations that accompany resistance are indicated. 
resistant MCF7-ARO cells. As expected, the elevated phospho-ER $\alpha^{\mathrm{Ser} 167}$ was diminished with taselisib treatment in the resistant cells. Interestingly, ER $\alpha$ levels did not correspond with a proliferative response to estrogen in our models. While the parental line exhibited a dose-response to added estrogen or androstenedione, letrozole resistant cells did not increase growth with either agent (Figure 3D).

We also investigated commonly described mechanisms of endocrine therapy resistance, such as MET and RET tyrosine kinases and FOSL1 transcriptional regulator [31, 32, 33]. Although we found the expression of some of these markers to be changed, using small molecule inhibitor and RNA interference knockdown studies we could not validate their involvement in resistance to endocrine therapies (Supplementary Figure 4).

Given that letrozole resistant MCF7-ARO models remained sensitive to taselisib, treatment of endocrine resistant tumors with taselisib may be appropriate and offer clinical benefit. Hence, we next wanted to model the possibility of eventual progression following the treatment of letrozole resistant cells with taselisib. Letrozole-R1 and $-\mathrm{R} 2$ cells were treated at increasing doses of taselisib over a period of 8 months. At the end of the dose escalation, the cells were able to grow in the presence of taselisib at a concentration greater than 25 -fold higher $(2.5 \mu \mathrm{M})$ than the initial $\mathrm{EC}_{50}$ dose $(0.09 \mu \mathrm{M})$. The newly generated taselisib-resistant pools also remained highly resistant to letrozole, compared to the parental MCF7-ARO line (Figure 4A).

Once the pools were confirmed to be dually resistant to letrozole and taselisib, signaling components of the PI3K and ER pathways were evaluated by western blot analysis in comparison to parental and letrozole resistant pools. The elevated levels of $\mathrm{pAkt}^{\mathrm{S} 473}, \mathrm{ER} \alpha$ and $\mathrm{pER} \alpha^{\mathrm{S} 167}$ that were observed in letrozole resistant cells were reduced in the dually resistant cells to levels comparable to those of MCF7-ARO parental cells (Figure 4B). The letrozole resistant and dual resistant pools both had reduced PR compared to the parental cells.

\section{Cells resistant to both letrozole and taselisib remain sensitive to taselisib in combination with docetaxel or CDK4/6 inhibition}

We sought to evaluate if letrozole resistant and dual letrozole/taselisib resistant cells were sensitive to other inhibitors that are being used to treat ER positive breast cancer patients [34]. Overall, we found that the parental and letrozole resistant cells remained sensitive to docetaxel and the CDK4/6 inhibitor PD-0332991, but these agents had reduced potency in dual resistant clones (Figure 5A and 5B).

To evaluate docetaxel in combination with taselisib, the MCF7-ARO parental, letrozole-R1 and Let-R1. GDC-0032-R dual resistant cells were treated with these agents in a dose titration for each line (Figure 5A). In these experiments, drug concentrations were the same across the cell lines. Decreased viability was observed relative to single agent treatments in each cell line model. Interestingly, docetaxel did not have an effect in the dual resistant cells as a single agent, but was effective in combination with taselisib.

The effect of the compounds on downstream PI3K pathway markers Akt and S6, the apoptotic marker cleaved PARP, and cyclin D1 were investigated with single agent and combination drug treatments at $\mathrm{EC}_{50}$ concentrations for the parental cell line at a 24-hour timepoint (Figure $5 \mathrm{C})$. In the drug combination increased cleaved PARP and a small reduction in cyclin D1 was observed in the three cell line models. As expected, decreases in PI3K pathway signaling were observed with taselisib treatments.

We also evaluated PD-0332991 in combination with taselisib in parental and resistant clones (Figure 5B). PD-0332991 (Palbociclib) is a CDK4/6 small molecule inhibitor currently under evaluation in the clinic for ER+ breast cancer in combination with letrozole [34]. Relative to single agent treatments, decreased viability was observed with the PD-0332991 and taselisib combination in each cell line model similar to findings in other studies [35].

To determine the effects of the PD-0332991 and taselisib drug combination in parental and resistant cell line models, we assessed cyclin D1, cyclin E, phosphorylated $\mathrm{Rb}$ (Ser807/811) and cleaved PARP after 24 hours of treatment (Figure 5D). Cleaved PARP was detected with all taselisib treatments and a decrease in cyclin $\mathrm{E}$ was detected with the drug combination. Hyperphosphorylation of $\mathrm{Rb}$ at multiple sites, including 807 and 811 is indicative of cells that have entered the cell cycle and are proliferating. Both letrozole and dual letrozole/taselisib resistant cells had increased phosphorylation of $\mathrm{Rb}^{\text {Ser807/811 }}$ that was decreased with PD0332991 and taselisib combination drug treatment. This molecular mechanism is consistent with a recent report using additional PI3K and CDK4/6 inhibitors with MCF7 and T47D parental cells [35]. As expected, decreases in PI3K pathway signaling were observed with taselisib treatments.

\section{DISCUSSION}

Breast cancer is a molecularly and clinically heterogeneous disease [36] and there remains a significant unmet medical need. Patients with ER-positive/HER2negative tumors have clinical benefit when receiving hormone therapies, although recurrence can occur despite adjuvant endocrine treatment. Prolonged inhibition of ER signaling has been evaluated and several clinical trials have demonstrated the value of extended use of 
aromatase inhibitors, such as letrozole, following 5 years of tamoxifen treatment [37]. Another approach to address endocrine therapy resistance is to target the signaling crosstalk between ER and other pathways. Given that components of the PI3K pathway, and specifically the $P I K 3 C A$ gene, are frequently mutated in breast cancer (77\% and $35 \%$, respectively) [38] and that crosstalk between ER and PI3K signaling pathways has been established [17, 39], we asked whether PI3K inhibition could decrease proliferation of breast cancer cells in the context of resistance to aromatase inhibition.

In our studies the PI3K inhibitor taselisib potently inhibited PI3K pathway signaling and combined well with letrozole in an aromatase-expressing breast cancer cell line (Figure 1). Our studies demonstrate that increased PI3K pathway activation confers resistance to letrozole. In a secreted factor screen, multiple soluble factors lead to letrozole resistance and these factors also increase PI3K signaling (Figure 2). PI3K pathway activity was also elevated in cells that were selected for acquired letrozole resistance, but the increased signaling could be reduced by taselisib treatment (Figure 3A). Moreover, under these conditions of acquired letrozole resistance the tumor cells were equally sensitive to taselisib. Letrozole-resistant cells expressed higher $\mathrm{p} 110 \alpha$ protein levels, which may be responsible for the increase in PI3K pathway signaling (Figure 4B). Importantly, although letrozole resistant cells had increased expression levels of ER, these tumor lines did not utilize estrogen for growth and had decreased amounts of the ER target gene PGR, further suggesting that they were using alternative pathways for growth (Figure 4B).

The letrozole resistant cells were used to create a breast cancer cell line model that was resistant to both taselisib and letrozole (Figure 4). Interestingly, the elevated $\mathrm{p} 110 \alpha$ and ER proteins observed in the letrozole resistant cells had returned to parental levels in the dual resistant cells. Despite resistance to letrozole or taselisib, the cells were sensitive to taselisib in combination with other ER+ breast cancer therapies, docetaxel and the PD0332991 CDK4/6 inhibitor. Taken together, these data provide further rationale for evaluating PI3K pathway inhibitors for $\mathrm{HR}+$ breast cancer treatment in the clinic (Figure 6).

Everolimus, an inhibitor of mTOR (which is a critical component of the PI3K pathway), has been shown to prolong progression-free survival in combination with the aromatase inhibitor, exemestane [40]. Encouraged by the efficacy and safety detected with concomitant inhibition of mTOR and ER signaling, a number of novel agents that target the PI3K pathway are currently in clinical trials, including the novel PI3K inhibitor, taselisib. In these cell line models of letrozole resistance, taselisib treatment alone or in combination with other therapies was able to re-sensitize breast cancer resistant models.

\section{MATERIALS AND METHODS}

\section{Cell culture}

MCF7 cell line was obtained from the American Type Culture Collection (ATCC, VA). The cells were tested and authenticated using gene expression and single nucleotide polymorphism genotyping arrays, as previously described [41, 42] and cultured in RPMI supplemented with $10 \%$ fetal bovine serum, 100 units $/ \mathrm{ml}$ penicillin, 100 $\mu \mathrm{g} / \mathrm{ml}$ streptomycin, $2 \mathrm{mM}$ L-glutamine and NEAA at $37^{\circ} \mathrm{C}$ under $5 \% \mathrm{CO}_{2}$. Stable aromatase-expressing MCF7 cells (MCF7-ARO) were generated by transfection of a plasmid vector containing the full aromatase gene and a neomycin selection gene. The cells were maintained in androstenedione and all experiments were performed in the presence of androstenedione except where indicated (Figure 3D).

\section{Materials}

Taselisib, also called GDC-0032, was generated at Genentech, Inc. (South San Francisco, CA). Letrozole was obtained from US Biological. Antibodies used include phospho-AKT ${ }^{\mathrm{Ser} 473}$, AKT, phospho-PRAS40 ${ }^{\text {Thr246, }}$ phospho-S6 ${ }^{\mathrm{Ser} 235 / 236}$, phospho-S6 $6^{\mathrm{Ser} 240 / 242}$, S6, phospho$\mathrm{ERK}^{\mathrm{Thr202/Tyr204}}$, ERK, phospho-ER $\alpha^{\mathrm{Ser118}}$, phospho$\mathrm{ER} \alpha^{\mathrm{Ser} 167}$, cleaved PARP, p110 $\alpha$, phospho-p70S6K ${ }^{\mathrm{Th} 389}$,

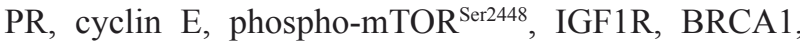
c-Myc, CAV1, HER2 and cyclin D1 obtained from Cell Signaling (Danvers, MA). Antibodies for ER $\alpha$ and ER $\beta$ were obtained from Santa Cruz biotechnology (Santa Cruz, CA) and a $\beta$ Actin antibody was obtained from Sigma (St. Louis, MO).

\section{Cell viability assays}

384-well plates were seeded with 2000 cells/well in a volume of $54 \mu \mathrm{l}$ per well followed by incubation at $37^{\circ} \mathrm{C}$ under $5 \% \mathrm{CO}_{2}$ overnight ( $\sim 16$ hours). Compounds were diluted in DMSO to generate the desired stock concentrations then added in a volume of $6 \mu \mathrm{L}$ per well. All treatments were tested in quadruplicate. After 4 days incubation, relative numbers of viable cells were estimated using CellTiter-Glo (Promega, Madison, WI) and total luminescence was measured on an Envision plate Reader (PerkinElmer, Foster City,CA). The concentration of drug resulting in $50 \%$ inhibition of cell viability $\left(\mathrm{IC}_{50}\right)$ or $50 \%$ maximal effective concentration $\left(\mathrm{EC}_{50}\right)$ was determined using Prism software (GraphPad, La Jolla, CA). For cell lines that failed to achieve an $\mathrm{EC}_{50}$ the highest concentration tested $(10 \mu \mathrm{M})$ is listed. 


\section{Letrozole resistant cell line selection}

MCF7-ARO cells were grown in increasing concentrations of letrozole in the presence of androstenedione in phenol red free RPMI medium, supplement with $10 \%$ Charcoal dextran stripped FBS, until they grew normally in a letrozole concentration of $6.5 \mu \mathrm{mol} / \mathrm{L}$. For cells resistant to both letrozole and taselisib, letrozole resistant cells were grown in increasing concentrations of the taselisib, until they grew normally in a concentration of $2.5 \mu \mathrm{mol} / \mathrm{L}$. Maintenance of aromatase expression in all letrozole sensitive and resistant clones was verified using TaqMan.

\section{Protein assays}

$10 \mathrm{~cm}^{2}$ dishes were seeded with two million cells in a volume of $10 \mathrm{~mL}$ followed by incubation at $37^{\circ} \mathrm{C}$ under $5 \% \mathrm{CO}_{2}$ overnight ( $\sim 16$ hours). Cells were treated with the indicated concentration of taselisib or pictilisib for the time indicated. Following treatment, cells were washed with cold PBS and lysed in 1X Cell Extraction Buffer from Invitrogen (Carlsbad, CA) supplemented with protease inhibitors (Roche, Germany), Phosphatase Inhibitor Cocktails 2 and 3 from Sigma (St. Louis, MO). Protein concentration was determined using the Pierce BCA Protein Assay Kit (Rockford, IL). For immunoblots, equal protein amounts were separated by electrophoresis through NuPage Bis-Tris 4-12\% gradient gels (Invitrogen, Carlsbad, CA); proteins were transferred onto nitrocellulose membranes using the iBlot system from Invitrogen (Carlsbad, CA).

\section{Secreted factor screen}

Recombinant purified secreted factors were purchased from Peprotech and R\&D Systems as indicated, and were reconstituted in PBS/0.1\% BSA (Supplementary Table 1). Secreted factors were transferred into 96-well plates at a concentration of $1 \mu \mathrm{g} / \mathrm{ml}$, and subsequently diluted to $100 \mathrm{ng} / \mathrm{ml}$ in media containing either no drug or $0.6 \mu \mathrm{M}$ of taselisib or $1 \mu \mathrm{M}$ letrozole. Equal volumes of diluted factor (final concentration $50 \mathrm{ng} / \mathrm{ml}$ ) were arrayed into the 384 well plates pre-seeded with cells (2000 cells per wells seeded the day before) using a Bravo liquid handler. After 72 hours incubation, cell viability was determined using CellTiter-Glo (Promega).

\section{Statistics}

Significant differences comparing lines with and without evaluated genetic abnormalities was determined by two-tailed Mann-Whitney test calculated using the JMP statistical software, version 5.1.2 and p values reported (JMP Software, Cary, NC).

\section{CONCLUSIONS}

The results of this study suggest that selective PI3K inhibition, either alone or in combination with other breast cancer treatment modalities, may be efficacious in HR+ tumors that are either sensitive or refractory to single agent endocrine therapy such as letrozole treatment.

\section{ABBREVIATIONS}

PI3K: phosphoinositide-3 kinase; HR: hormone receptor; ER: estrogen receptor; PR: progesterone receptor; PGR: progesterone receptor gene; CDK: cyclin dependent kinase; EC: effective concentration; IC: inhibitory concentration; OSM: oncostatin M; LTED: long term estrogen deprived

\section{ACKNOWLEDGEMENTS}

The authors wish to thank Genentech chemists for taselisib.

\section{CONFLICTS OF INTERESTS}

All authors are employees of Genentech, Inc.

\section{AUTHOR'S CONTRIBUTIONS}

$\mathrm{KPH}$ conceived and designed experiments and helped to draft the manuscript. JG carried out the immunoblots and cell viability experiments. KAE designed and carried out cell viability experiments. $\mathrm{CO}$, HS, TRW, and RMN carried out the secreted factor screen. LSF participated in the design of the study and helped to revise the manuscript. JJW conceived of the study, and participated in its design and coordination and drafted the manuscript. All authors read and approved the final manuscript.

\section{REFERENCES}

1. Jemal A, Bray F, Center MM, Ferlay J, Ward E, Forman D. Global cancer statistics. CA Cancer J Clin. 2011;61(2):6990. Epub 2011/02/08. doi: caac.20107 [pii] 10.3322/ caac.20107. PubMed PMID: 21296855.

2. Colditz GA. Relationship between estrogen levels, use of hormone replacement therapy, and breast cancer. J Natl Cancer Inst. 1998;90(11):814-23. Epub 1998/06/13. PubMed PMID: 9625169.

3. Hankinson SE, Colditz GA, Willett WC. Towards an integrated model for breast cancer etiology: the lifelong 
interplay of genes, lifestyle, and hormones. Breast Cancer Res. 2004;6(5):213-8. Epub 2004/08/21. doi: 10.1186/ bcr921 bcr921 [pii]. PubMed PMID: 15318928; PubMed Central PMCID: PMC549181.

4. Howell A. The endocrine prevention of breast cancer. Best Pract Res Clin Endocrinol Metab. 2008;22(4):615-23. Epub 2008/10/31. doi: S1521-690X(08)00100-0 [pii] 10.1016/j. beem.2008.09.002. PubMed PMID: 18971122.

5. Cuzick J. Aromatase inhibitors for breast cancer prevention. J Clin Oncol. 2005;23(8):1636-43. Epub 2005/03/10. doi: 23/8/1636 [pii] 10.1200/JCO.2005.11.027. PubMed PMID: 15755971 .

6. Howell A, Cuzick J, Baum M, Buzdar A, Dowsett M, Forbes JF, et al. Results of the ATAC (Arimidex, Tamoxifen, Alone or in Combination) trial after completion of 5 years' adjuvant treatment for breast cancer. Lancet. 2005;365(9453):60-2. Epub 2005/01/11. doi: S0140673604176666 [pii] 10.1016/S0140-6736(04)176666. PubMed PMID: 15639680.

7. Yuan TL, Cantley LC. PI3K pathway alterations in cancer: variations on a theme. Oncogene. 2008;27(41):5497510. Epub 2008/09/17. doi: onc2008245 [pii] 10.1038/ onc.2008.245. PubMed PMID: 18794884.

8. Bader AG, Kang S, Zhao L, Vogt PK. Oncogenic PI3K deregulates transcription and translation. Nat Rev Cancer. 2005;5(12):921-9. PubMed PMID: 16341083.

9. Samuels Y, Wang Z, Bardelli A, Silliman N, Ptak J, Szabo $\mathrm{S}$, et al. High frequency of mutations of the PIK3CA gene in human cancers. Science. 2004;304(5670):554. PubMed PMID: 15016963.

10. Zhao L, Vogt PK. Class I PI3K in oncogenic cellular transformation. Oncogene. 2008;27(41):5486-96. Epub 2008/09/17. doi: onc2008244 [pii] 10.1038/onc.2008.244. PubMed PMID: 18794883.

11. Zhang S, Yu D. PI(3)king apart PTEN's role in cancer. Clin Cancer Res. 2010;16(17):4325-30. Epub 2010/07/14. doi: 1078-0432.CCR-09-2990 [pii] 10.1158/1078-0432.CCR09-2990. PubMed PMID: 20622047.

12. Klempner SJ, Costa DB, Wu PA, Ariyabuddhiphongs KD. Safety of cupping during bevacizumab therapy. J Altern Complement Med. 2013;19(8):729-31. doi: 10.1089/ acm.2011.0791. PubMed PMID: 23379829.

13. Folkes AJ, Ahmadi K, Alderton WK, Alix S, Baker SJ, Box $\mathrm{G}$, et al. The identification of 2-(1H-indazol-4-yl)-6-(4methanesulfonyl-piperazin-1-ylmethyl)-4-morpholin -4-ylthieno[3,2-d]pyrimidine (GDC-0941) as a potent, selective, orally bioavailable inhibitor of class I PI3 kinase for the treatment of cancer. J Med Chem. 2008;51(18):552232. Epub 2008/08/30. doi: 10.1021/jm800295d. PubMed PMID: 18754654.

14. Maira SM, Stauffer F, Brueggen J, Furet P, Schnell C, Fritsch $\mathrm{C}$, et al. Identification and characterization of NVPBEZ235, a new orally available dual phosphatidylinositol 3-kinase/mammalian target of rapamycin inhibitor with potent in vivo antitumor activity. Mol Cancer Ther. 2008;7(7):1851-63. Epub 2008/07/09. doi: 1535-7163. MCT-08-0017 [pii] 10.1158/1535-7163.MCT-08-0017. PubMed PMID: 18606717.

15. Liu P, Cheng H, Roberts TM, Zhao JJ. Targeting the phosphoinositide 3-kinase pathway in cancer. Nat Rev Drug Discov. 2009;8(8):627-44. Epub 2009/08/01. doi: nrd2926 [pii] 10.1038/nrd2926. PubMed PMID: 19644473.

16. Ndubaku CO, Heffron TP, Staben ST, Baumgardner $\mathrm{M}$, Blaquiere $\mathrm{N}$, Bradley $\mathrm{E}$, et al. Discovery of 2-\{3-[2-(1-Isopropyl-3-methyl-1H-1,2-4-triazol-5-yl)-5,6dihydrobenzo[f]imidazo[1 ,2-d][1,4] oxazepin-9-yl]-1H-

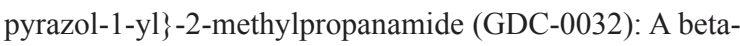
Sparing Phosphoinositide 3-Kinase Inhibitor with High Unbound Exposure and Robust in Vivo Antitumor Activity. J Med Chem. 2013;56(11):4597-610. Epub 2013/05/15. doi: 10.1021/jm4003632. PubMed PMID: 23662903.

17. Bosch A, Li Z, Bergamaschi A, Ellis H, Toska E, Prat A, et al. PI3K inhibition results in enhanced estrogen receptor function and dependence in hormone receptor-positive breast cancer. Sci Transl Med. 2015;7(283):283ra51. doi: 10.1126/scitranslmed.aaa4442. PubMed PMID: 25877889; PubMed Central PMCID: PMCPMC4433148.

18. Fox EM, Arteaga CL, Miller TW. Abrogating endocrine resistance by targeting ERalpha and PI3K in breast cancer. Front Oncol. 2012;2:145. Epub 2012/10/23. doi: 10.3389/ fonc.2012.00145. PubMed PMID: 23087906; PubMed Central PMCID: PMC3472546.

19. Boulay A, Rudloff J, Ye J, Zumstein-Mecker S, O'Reilly T, Evans DB, et al. Dual inhibition of mTOR and estrogen receptor signaling in vitro induces cell death in models of breast cancer. Clin Cancer Res. 2005;11(14):5319-28. Epub 2005/07/22. doi: 10.1158/1078-0432.CCR-04-2402. PubMed PMID: 16033851.

20. Oliver FJ, de la Rubia G, Rolli V, Ruiz-Ruiz MC, de Murcia G, Murcia JM. Importance of poly(ADP-ribose) polymerase and its cleavage in apoptosis. Lesson from an uncleavable mutant. J Biol Chem. 1998;273(50):33533-9. Epub 1998/12/05. PubMed PMID: 9837934.

21. Campbell RA, Bhat-Nakshatri P, Patel NM, Constantinidou D, Ali S, Nakshatri H. Phosphatidylinositol 3-kinase/ AKT-mediated activation of estrogen receptor alpha: a new model for anti-estrogen resistance. J Biol Chem. 2001;276(13):9817-24. Epub 2001/01/15. doi: 10.1074/jbc. M010840200. PubMed PMID: 11139588.

22. Joel PB, Smith J, Sturgill TW, Fisher TL, Blenis J, Lannigan DA. pp90rsk1 regulates estrogen receptor-mediated transcription through phosphorylation of Ser-167. Mol Cell Biol. 1998;18(4):1978-84. Epub 1998/04/07. PubMed PMID: 9528769; PubMed Central PMCID: PMC121427.

23. Lippman ME, Rae JM, Chinnaiyan AM. An expression signature of estrogen-regulated genes predicts diseasefree survival in tamoxifen-treated patients better than progesterone receptor status. Trans Am Clin Climatol Assoc. 2008;119:77-90; discussion -2. Epub 2008/07/04. 
PubMed PMID: 18596862; PubMed Central PMCID: PMC2442700.

24. Oh DS, Troester MA, Usary J, Hu Z, He X, Fan C, et al. Estrogen-regulated genes predict survival in hormone receptor-positive breast cancers. J Clin Oncol. 2006;24(11):1656-64. Epub 2006/03/01. doi: 10.1200/ JCO.2005.03.2755. PubMed PMID: 16505416.

25. van 't Veer LJ, Dai H, van de Vijver MJ, He YD, Hart AA, Mao M, et al. Gene expression profiling predicts clinical outcome of breast cancer. Nature. 2002;415(6871):530-6. Epub 2002/02/02. doi: 10.1038/415530a. PubMed PMID: 11823860 .

26. Ibrahim YH, Garcia-Garcia C, Serra V, He L, TorresLockhart K, Prat A, et al. PI3K inhibition impairs BRCA1/2 expression and sensitizes BRCA-proficient triple-negative breast cancer to PARP inhibition. Cancer Discov. 2012;2(11):1036-47. Epub 2012/08/24. doi: 10.1158/21598290.CD-11-0348. PubMed PMID: 22915752.

27. Chen D, Riedl T, Washbrook E, Pace PE, Coombes RC, Egly JM, et al. Activation of estrogen receptor alpha by S118 phosphorylation involves a ligand-dependent interaction with TFIIH and participation of CDK7. Mol Cell. 2000;6(1):127-37. Epub 2000/08/19. PubMed PMID: 10949034.

28. Hintzen C, Haan C, Tuckermann JP, Heinrich PC, Hermanns HM. Oncostatin M-induced and constitutive activation of the JAK2/STAT5/CIS pathway suppresses CCL1, but not CCL7 and CCL8, chemokine expression. J Immunol. 2008;181(10):7341-9. Epub 2008/11/05. PubMed PMID: 18981157.

29. Miller TW, Balko JM, Fox EM, Ghazoui Z, Dunbier A, Anderson $\mathrm{H}$, et al. ERalpha-dependent E2F transcription can mediate resistance to estrogen deprivation in human breast cancer. Cancer Discov. 2011;1(4):338-51. Epub 2011/11/04. doi: 10.1158/2159-8290.CD-11-0101. PubMed PMID: 22049316; PubMed Central PMCID: PMC3204388.

30. Miller TW, Hennessy BT, Gonzalez-Angulo AM, Fox EM, Mills GB, Chen H, et al. Hyperactivation of phosphatidylinositol-3 kinase promotes escape from hormone dependence in estrogen receptor-positive human breast cancer. J Clin Invest. 2010;120(7):2406-13. Epub 2010/06/10. doi: 10.1172/JCI41680. PubMed PMID: 20530877; PubMed Central PMCID: PMC2898598.

31. Morandi A, Martin LA, Gao Q, Pancholi S, Mackay A, Robertson D, et al. GDNF-RET signaling in ERpositive breast cancers is a key determinant of response and resistance to aromatase inhibitors. Cancer Res. 2013;73(12):3783-95. Epub 2013/05/08. doi: 10.1158/00085472.CAN-12-4265. PubMed PMID: 23650283; PubMed Central PMCID: PMC3686594.

32. Nehra R, Riggins RB, Shajahan AN, Zwart A, Crawford AC, Clarke R. BCL2 and CASP8 regulation by NF-kappaB differentially affect mitochondrial function and cell fate in antiestrogen-sensitive and -resistant breast cancer cells. FASEB J. 2010;24(6):2040-55. Epub 2010/02/16. doi: 10.1096/fj.09-138305. PubMed PMID: 20154269; PubMed Central PMCID: PMC2874480.

33. Pennanen PT, Sarvilinna NS, Toimela T, Ylikomi TJ. Inhibition of FOSL1 overexpression in antiestrogenresistant MCF-7 cells decreases cell growth and increases vacuolization and cell death. Steroids. 2011;76(10-11):1063-8. Epub 2011/05/17. doi: 10.1016/j. steroids.2011.04.011. PubMed PMID: 21570421.

34. Finn RS, Dering J, Conklin D, Kalous O, Cohen DJ, Desai AJ, et al. PD 0332991, a selective cyclin D kinase 4/6 inhibitor, preferentially inhibits proliferation of luminal estrogen receptor-positive human breast cancer cell lines in vitro. Breast Cancer Res. 2009;11(5):R77. Epub 2009/10/31. doi: 10.1186/bcr2419. PubMed PMID: 19874578; PubMed Central PMCID: PMC2790859.

35. Vora SR, Juric D, Kim N, Mino-Kenudson M, Huynh T, Costa C, et al. CDK 4/6 Inhibitors Sensitize PIK3CA Mutant Breast Cancer to PI3K Inhibitors. Cancer Cell. 2014;26(1):136-49. Epub 2014/07/09. doi: 10.1016/j. ccr.2014.05.020. PubMed PMID: 25002028.

36. Curtis C, Shah SP, Chin SF, Turashvili G, Rueda OM, Dunning MJ, et al. The genomic and transcriptomic architecture of 2,000 breast tumours reveals novel subgroups. Nature. 2012;486(7403):346-52. Epub 2012/04/24. doi: 10.1038/nature10983. PubMed PMID: 22522925; PubMed Central PMCID: PMC3440846.

37. Goss PE, Ingle JN, Martino S, Robert NJ, Muss HB, Piccart $\mathrm{MJ}$, et al. A randomized trial of letrozole in postmenopausal women after five years of tamoxifen therapy for early-stage breast cancer. N Engl J Med. 2003;349(19):1793-802. Epub 2003/10/11. doi: 10.1056/NEJMoa032312. PubMed PMID: 14551341 .

38. Comprehensive molecular portraits of human breast tumours. Nature. 2012;490(7418):61-70. Epub 2012/09/25. doi: 10.1038/nature11412. PubMed PMID: 23000897; PubMed Central PMCID: PMC3465532.

39. Schiff R, Massarweh SA, Shou J, Bharwani L, Mohsin SK, Osborne CK. Cross-talk between estrogen receptor and growth factor pathways as a molecular target for overcoming endocrine resistance. Clin Cancer Res. 2004;10(1 Pt 2):331S-6S. Epub 2004/01/22. PubMed PMID: 14734488.

40. Baselga J, Campone M, Piccart M, Burris HA, 3rd, Rugo HS, Sahmoud T, et al. Everolimus in postmenopausal hormone-receptor-positive advanced breast cancer. N Engl J Med. 2012;366(6):520-9. Epub 2011/12/14. doi: 10.1056/ NEJMoa1109653. PubMed PMID: 22149876.

41. Hoeflich KP, O'Brien C, Boyd Z, Cavet G, Guerrero $\mathrm{S}$, Jung $\mathrm{K}$, et al. In vivo antitumor activity of MEK and phosphatidylinositol 3-kinase inhibitors in basal-like breast cancer models. Clin Cancer Res. 2009;15(14):464964. Epub 2009/07/02. doi: 1078-0432.CCR-09-0317 [pii] 10.1158/1078-0432.CCR-09-0317. PubMed PMID: 19567590 .

42. Hu X, Stern HM, Ge L, O’Brien C, Haydu L, Honchell 
$\mathrm{CD}$, et al. Genetic alterations and oncogenic pathways associated with breast cancer subtypes. Mol Cancer Res. 2009;7(4):511-22. Epub 2009/04/18. doi: 7/4/511 [pii] 10.1158/1541-7786.MCR-08-0107. PubMed PMID: 19372580. 